\title{
Rayleigh Hysteresis Shape: Its Relationship to Displacement Distance of a Single Domain Wall in $50 \% \mathrm{Ni}-\mathrm{Fe}$
}

\author{
R. C. Woodbury \\ M. R. Hunt
}

Follow this and additional works at: https://scholarsarchive.byu.edu/facpub

Part of the Astrophysics and Astronomy Commons, and the Physics Commons

\section{Original Publication Citation}

Woodbury, R. C. and M. R. Hunt."Rayleigh Hysteresis Shape: Its Relationship to Displacement Distance of a Single Domain Wall in 5 Ni[Single Bond]Fe." Journal of Applied Physics 41 (197): 1159-116.

\section{BYU ScholarsArchive Citation}

Woodbury, R. C. and Hunt, M. R., "Rayleigh Hysteresis Shape: Its Relationship to Displacement Distance of a Single Domain Wall in 50\% Ni-Fe" (1970). Faculty Publications. 796.

https://scholarsarchive.byu.edu/facpub/796 


\title{
Domains
}

\author{
E. J. Torok, Chairman
}

\section{Rayleigh Hysteresis Shape: Its Relationship to Displacement Distance of a Single Domain Wall in $50 \% \mathrm{Ni}-\mathrm{Fe}^{*}$}

\author{
R. C. WOOdBury and M. R. Hunt $\dagger$ \\ Brigham Young Unizersity, Provo, Utah 84601
}

\begin{abstract}
The need to measure the displacement distance of domain walls from equilibrium for very weak alternating fields ( $H$ «coercive force) in bulk magnetic material has prompted a study of the possible use of the opening of the Rayleigh hysteresis loop to indicate the amount of wall displacement. This paper contains (1) a review of the theoretical relationship between hysteresis shape and the displacement distance of a domain wall, based upon defect-energy models of Rodbell and Bean, and Baldwin; and (2) an experimental approach which provides a measure of the wall displacements versus hysteresis shape for $\mathbf{5 0 \%} \mathrm{Ni}-\mathrm{Fe}$ tape. Discrepancies between the theoretical and measured relationships are noted. In addition, an application is described in which the measured relationship between hysteresis and wall displacement is used to obtain the size of residual domains in $50 \%$ nickeliron.
\end{abstract}

\section{INTRODUCTION}

Small displacements of domain walls in soft magnetic alloys and other materials are accompanied by nonconservative restoring forces which cause the so-called Rayleigh hysteresis ${ }^{1}$ loop to appear as shown in Fig. 1.

The usefulness of the Rayleigh hysteresis loop as a possible tool in measuring the displacement distance of domain walls in some uniform materials becomes apparent if, for calibration purposes, a known configuration of walls could be established. In addition, if some understanding of the mechanisms of Rayleigh hysteresis was available, one could choose that aspect or property of the loop which is most clearly allied with displacement distance.

The purpose of this paper is to demonstrate how the Rayleigh hysteresis is correlated with displacement distance for a particular material, $50 \% \mathrm{Ni}-\mathrm{Fe}$ tape; to compare this correlation with recent theoretical studies; and to illustrate, by example, the use of the hysteresis as a tool to measure displacements of unknown wall configurations.

Presented are (1) a theoretical relationship between the displacement distance of domain walls and the shape of the hysteresis based on the work of Baldwin ${ }^{2}$; (2) an experimental approach and a comparison with portions of Baldwin's theory; and (3) an example illustrating how the hysteresis opening, indicating distance of wall displacement, was used to indirectly determine the size of residual domains in $50 \% \mathrm{Ni}-\mathrm{Fe}$ tape.

\section{ANALYTICAL MODEL}

Baldwin ${ }^{2}$ has proposed a model in which the domain wall moves as a rigid membrane past defects exhibiting linear energy profiles and unequal distances of acquisition $\left(d_{a}\right)$ and release $\left(d_{r}\right)$. Such a model gives rise to a yield distance $\left(Z_{y}\right)$ for each family of defect sizes. When one considers the pressure acting to restore a wall to equilibrium, due to a given class of defect size, the product of defect density per unit size range $(n)$ and force per defect profile $(f)$ occurs as an important parameter. Assuming the product $n f$ to be a constant for all defect sizes out to a size having a yield distance $Z_{y m}$ (complete release), Baldwin has shown that the field on a wall moving between limits $+Z_{1}$ and $-Z_{1}$ is given by the expression:

$$
H(Z)=\left[Z\left(2 Z_{y m}-Z_{1}\right) \mp \frac{1}{2}\left(Z^{2}-Z_{1}^{2}\right)\right] n f /\left(2 M_{s}\right),
$$

where $Z$ is the distance from equilibrium. The negative

FIG. 1. Typical Rayleigh hysteresis

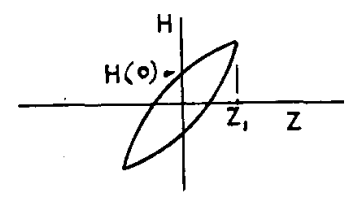

sign refers to the upper portion of the loop (wall moving in the positive $Z$ direction) and the positive sign the lower portion. Note that the peak restoring field, $H\left(Z_{1}\right)$, becomes

$$
H\left(Z_{1}\right)=\left[Z_{1}\left(2 Z_{y m}-Z_{1}\right)\right] n f /\left(2 M_{s}\right) .
$$

The field required to move the wall back through the equilibrium position $(Z=0)$ would be

$$
|H(0)|=\frac{1}{2} Z_{1}^{2} n f /\left(2 M_{8}\right) \text {. }
$$

If the assumption is made that all other forces on the domain wall, such as those arising from surface tension or magnetostatic energies, are conservative, then the only force acting on the wall at $Z=0$ would be of the nature of Eq. (3). Thus the field required to reach $Z=0$, after displacing the wall a peak distance $Z_{1}$, becomes the logical measure of peak displacement 1159 


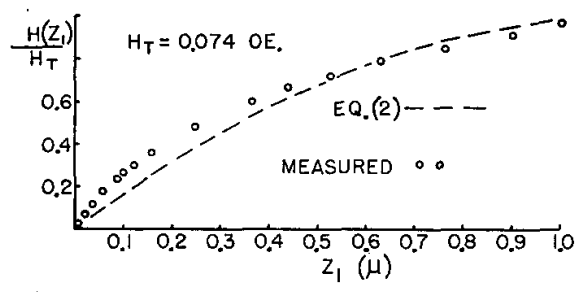

Fig. 2. Restoring field versus maximum displacement of walls. The experiment error is approximately $5 \%$.

distance. The experimental work described below was found to suggest that the theory is inadequate with the constant $n f$ function assumed.

\section{MEASUREMENTS AND COMPARISONS}

To establish a known configuration of domain walls, a single-layer toroidal tape core (102 $\mu$ thick, $6.3 \mathrm{~mm}$ wide, $50 \% \mathrm{Ni}-\mathrm{Fe}$ ) was partially switched with a $4-\mathrm{Oe}$ field pulse of $25-\mu \mathrm{sec}$ duration. The high field caused the formation of two planar walls at a depth of about $40 \mu$ from both surfaces. Such a switching process has been described by many investigators in a manner similar to that of Papoulis. ${ }^{3}$ With two known walls in the toroid, a small $100-\mathrm{Hz}$ field $\left(H<H_{c}\right)$ was then applied parallel to the walls. The maximum wall displacements were determined from a measurement of flux change $\Delta \phi$ and use of the expression $Z_{1}=\Delta \phi /\left(4 w B_{s}\right)$, where $w$ is the tape width.

Shown in Fig. 2 is the resulting measurement of normalized peak field $H\left(Z_{1}\right)$ versus measured displacement $Z_{1}$. The normalization factor $H_{T}$ was arbitrarily chosen to be the estimated threshold field for irreversible behavior $\left[Z_{y m}{ }^{2} n f /\left(2 M_{s}\right)\right]$. Shown on the plot is also Baldwin's expression for $H\left(Z_{1}\right)$, Eq. (2), with $Z_{y m}$ and $n f /\left(2 M_{s}\right)$ given the values $1.17 \mu$ and $0.054 \mathrm{Oe} / \mu^{2}$ to achieve a reasonable fit. The measured field $H(0)$ required to bring the wall back through equilibrium is shown in Fig. 3, along with a plot of Eq. (3). It is obvious that Baldwin's theory, with a constant nf function, is inadequate to predict all of the behavior; however, it appears that a better theoretical model would result if $n f$ were a decreasing function for increasing defect size $Z_{y}$.

An improved theoretical model for hysteresis will not be pursued at this time, but it should be borne in mind that the loop opening $B(0)$ is a more complex function

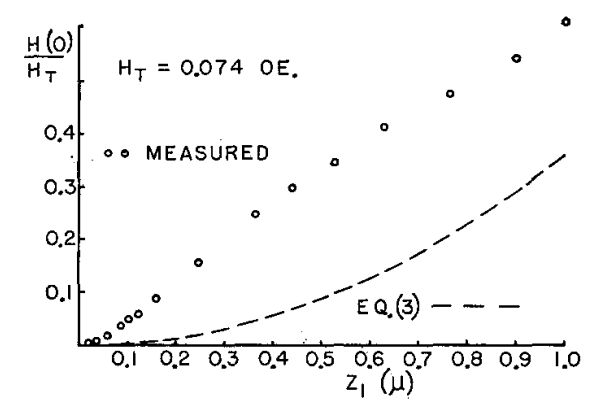

FIG. 3. Loop opening (field) versus maximum displacement of walls.

than Eq. (3); hence, one must use an experimental curve for $H(0)$ and not make a single-point evaluation of $n f$ for use in Eq. (3).

The principal observation to be made at this time, however, is that the actual $H(0)$ has been obtained as a function of $Z_{1}$; hence, displacement $Z_{1}$ of unknown configurations of walls can be found by measuring only $H(0)$. (This assumes that all new forces are conservative and only one type of domain is present.)

\section{EXAMPLE ${ }^{4}$}

The average size of residual domains, existing at saturation remanence in a closed-loop core of $50 \%$ $\mathrm{Ni}-\mathrm{Fe}$, was needed to correlate and predict the percentage of 2 nd harmonic flux resulting from a small sinusoidal field $\left(H<H_{c}\right)$. For spike-shaped domains, one can show that the ratio of 2 nd harmonic to fundamental components of flux should be $Z_{1} /\left(2 r_{0}\right)$, where $r_{0}$ is the equilibrium radius of the residual domain; hence, measurement of the flux signals along with $H(0)\left(Z_{1}\right.$ from Fig. 3) provides a calculation of $r_{0}$. The values for $r_{0}$ found by this method (2.5 to $6 \mu$ in core thicknesses between 25 and $100 \mu$ ) are in agreement with other estimations such as proposed by Leliakov. ${ }^{5}$

* Research partially supported by National Science Foundation Grant GK3395.

$\uparrow$ Present address: Motorola Semiconductor Products, Phoenix, Ariz.

${ }^{2} \mathrm{~A}$ characteristic $B-H$ loop occurring with very weak fields and named after the early investigator Lord Rayleigh.

${ }^{2}$ J. A. Baldwin, Jr., J. Appl. Phys. 38, 501 (1967).

3 A. Papoulis, J. Appl. Phys. 25, 169 (1954).

${ }^{4}$ R. C. Woodbury, Ph.D. dissertation, Stanford University, 1965.

${ }^{5}$ F. J. Friedlaender and I. P. Leliakov, AIEE Trans. (Commun. Electron.) 80, 402 (1961). 